\title{
Editorial
}

\section{Scientific publication in the digital era}

\author{
Bhaven C. Kataria* \\ Department of Pharmacology, GMERS Medical College, Sola, Ahmedabad, Gujarat, India \\ *Correspondence: \\ Dr. Bhaven C. Kataria, \\ E-mail:drbkataria@gmail.com
}

Scientific publication is undergoing a drastic transformation over the last decade. Now there is a trend of digitalization of all forms of communication. ${ }^{1,2}$ Few years ago authors were submitting their manuscripts in hard copies. Now most of the scientific journals are using online platform for the entire publishing process (i.e. manuscript submission, review, revision, proofs, publishing). Many traditional print journals are now using online platform as there is an increase in usage of digital devices (computer, laptop, tablet or smartphone) by scientists and clinicians to search online and read articles in digital form.

The key challenge of print based journals is the limited space available. Print journals are limited in space due to the high cost of printing and distributing. Therefore, they include only the minimum information essential to understand the research work. There is always words limit for each type of article and acceptability of article is low. Therefore, rejection rate is very high in high quality print journal. There is a possibility that your paper gets rejected not because of quality of research work, but it may be because of lack of space in the journal. There is a tough competition to get published in the high quality print journal. Graphics and photos are kept to a minimum in print journals, and color is almost rarely used.

One of the significant advantages of electronic form of the journal from author's point of view is the speed of publication. It's possible to publish articles rapidly in electronic form of publication. The articles can be posted online as they are ready (as online first) after peer review and proof checking. One element that we all love about digital information is its easy searchability. Accessibility is also very high with electronic form of the journal as your article is available for view and downloads within minutes anywhere in the world. Your article is available anywhere at anytime, you just need an internet connection.
The electronic age has opened the possibility of multiple data formats (e.g. html, pdf, epub, mobile html) for an enhanced reading experience. The storage space needed to preserve print materials is quite large. With electronic publishing, you can just store what you need in the cloud (Dropbox, OneDrive, Google Drive etc.) or in the storage space your digital device of choice can support. You can bring your collection of references anywhere you like hassle-free. There are now web based platforms like EndNote, Mendeley, RefWorks and Zotero where you can store your references. These are basically citation management tools, but they provide other functions as well. Mobile and tablet applications are available for some of the tools, so you can use on smartphones and tablets also.

The electronic journals should have emergency preservation strategy in place. If digital file is lost, due to a system failure or human error, and no reliable backup exists, all of your work could simply disappear. Scientific journal articles are some of the most intellectually valuable content that our society produces and so they should be appropriately preserved and protected from loss. To help online journal publishers overcome this potential disaster, Stanford University developed the open source LOCKSS project (Lots of Copies Keeps Stuff Safe). ${ }^{3}$ LOCKSS consists of geographically distributed servers maintained by libraries, the traditional experts in information storage and preservation. LOCKSS ensures that multiple copies of your content exists on a network of servers, that all of your latest content is collected and securely stored by a specialized web crawler. ${ }^{3}$ Reputable publishers of open-access journals arrange for their content to be archived in welltrusted third-party archives such as LOCKSS, CLOCKSS, Portico, PMC/Europe PMC/PMC Canada. With digital archiving, you can be much more confident that journal's content will still be around in 50, 100 and many years, even if publisher goes bankrupt, your article 
will be preserved in these archives if publisher has digital archiving policy in place.

The electronic form of the journals has capability to measure the impact of article by providing article tools. Some journals are adopting article based metrics as an alternative to citation-based impact factors, using the number of views/downloads of the article. Journal's impact factor and citations are common parameter of a journal or an article's influence. However, they don't fully capture how much your research might influence your field and related disciplines. Some journal publishers provide citation alert service where author gets e-mail whenever author's article is cited. There are some open-source, web-based tool (e.g. Impactstory) that helps researchers explore and share the diverse impacts of all their research work. ${ }^{4}$

It is very crucial that any research should be linked to their associated research articles as standard practice. Citations of relevant works are an integral part of all scientific research papers. Most journal articles can now be uniquely identified by permanent identifier such as digital object identifier (DOI). DOIs for journal articles are issued by CrossRef. There are also other unique identifiers for scholarly content, e.g. the PubMed ID, PubMed Central (PMC) ID, Handles, ARK, or EzID. Many publishers provide facility of citation reference linking. Citation references to full-text articles or abstract can be linked at another publisher's site, implemented through digital object identifiers (DOIs) managed through the CrossRef linking service. Many other links like PubMed, PMC or links to web resources such as technical reports can be found in references section. It's very crucial that authors should provide accurate references as per journal's style and format to reduce the errors in linking of references.

Beyond saving trees, scientific publication in the digital form means faster communication with the world, new level of accuracy, preserves your work for long time, less space is required to archive, link with similar research or can easily measure the impact of your research work. Whatever medium we use to publish our research work, one thing we should keep in mind that technology should not drive us. We should use technology to improve patient's health. That's our ultimate goal in medical profession.

\section{REFERENCES}

1. Topol E. The Creative Destruction of Medicine: How the Digital Revolution Will Create Better Health Care. New York: Basic Books; 2012.

2. De Groote SL. Citation patterns of online and print journals in the digital age. J Med Libr Assoc 2008;96:362-9.

3. LOCKSS. How LOCKSS Works. Available at http://www.lockss.org/about/how-it-works/. Accessed 9 March 2014.

4. Impactstory. Available at https://www.impactstory.org. Accessed 9 March 2014.

DOI: $10.5455 / 2349-2902 . i s j 20140501$

Cite this article as: Kataria BC. Scientific publication in the digital era. Int Surg J 2014;1:1-2. 